\title{
Pedestrian Evacuation Time Model for Urban Metro Hubs Based on Multiple Video Sequences Data
}

\author{
Ji-biao Zhou, ${ }^{1}$ Hong Chen, ${ }^{1,2}$ Jing Yang, ${ }^{1}$ and Jiao Yan ${ }^{1}$ \\ ${ }^{1}$ School of Highway, Chang'an University, Xian 710064, China \\ ${ }^{2} 902$ Transportation Science \& Technology Building, Chang'an University, Middle Section of Nanerhuan Road, Xi'an 710064, China \\ Correspondence should be addressed to Hong Chen; hongchen82@126.com
}

Received 25 November 2013; Accepted 23 January 2014; Published 10 March 2014

Academic Editor: Wuhong Wang

Copyright (c) 2014 Ji-biao Zhou et al. This is an open access article distributed under the Creative Commons Attribution License, which permits unrestricted use, distribution, and reproduction in any medium, provided the original work is properly cited.

\begin{abstract}
Evacuation time is a significant safety coefficient for Urban Metro Hubs (UMHs). Usually, a reasonable model for evacuation time will effectively promote the safety for pedestrian when emergency incidents occur in UMHs. In this paper, we propose a pedestrian evacuation time model for UMHs to improve the accuracy and reliability of its evacuation time. Firstly, we design an experiment survey based on the multiple video sequences to analyze the characteristics of pedestrian flow. Then, we decompose the evacuation process on the basis of the parameters, which involve the evacuation characteristics, the speed-density variation law, the pedestrian drop-off time, the platform evacuation time, and the channel evacuation time. Finally, we take the Bei Da-jie metro hub in Xi'an as an example, and verify the feasibility of the proposed pedestrian evacuation time model. The results show that the relative error for the evacuation time between the experiment result and the actual data is only $1.90 \%$, where the experiment time is $169.87 \mathrm{~s}$ and the actual time is $166.64 \mathrm{~s}$. Moreover, the proposed model strictly follows the Code for Design of Metro (GB 50157-003) and hence it can provide a good theoretical guidance for innovating the evacuation efficiency and the design reasonability of UMHs.
\end{abstract}

\section{Introduction}

Transport plays an important role in enhancing the quality of our living environment. The Urban Metro Hubs (UMHs), known as a key node of the integrated transport system, are the distribution center of passenger flow and play a crucial role in providing multimodal access to people and services in a manner that is convenient, safe, affordable, sustainable, efficient, and enjoyable. With the steady and rapid growth of passenger flow as well as the intensive transit, the closed environment space, and the complex transfer networks, the UMHs have become places vulnerable to extreme events, such as fire, stampede, and delay. Therefore, a time model for pedestrian evacuation is vital, for one thing, it can improve the efficiency of pedestrian evacuation, for another, it also ensure the passengers' life with a reasonable evacuation strategy.

In recent years, the pedestrian evacuation dynamics theory has become a hot topic in the academic field; they focus their attention on the passenger distribution characteristics and traffic behavior. It has made considerable progress since the 1980s; the researchers observed the characteristics of pedestrian movement regularity. With the deepening of the research on the regularity in the pedestrian movement, some also put physiological and psychological characteristics of pedestrians as a factor into consideration and set up a large amount of pedestrian simulation models, which is considered to be a powerful tool for evaluating pedestrian flows in facilities. These models can be classified as macroscopic and microscopic models. These scholars who study macroscopic models believe that pedestrian movement behavior is similar to the flow of gas or liquid [1-3]. The early macroscopic models include Game theory [4], Decision theory and Propagation model, Queuing model [5], Transformation Matrix model [6], Stochastic model [7], and Route Choice Behaviors model [8]. However, because the above models lack the consideration of the self-organizing behavior of pedestrians, these models do not have accurate prediction results. Therefore, some scholars turn their attention to microscopic pedestrian simulation models [9]. References [10-14] study normal state population self-organization phenomenon and 
proposed the social force model; many phenomena were simulated, which include the formation of the trail, oscillation at the bottleneck, and sheep-flock effect. Hereafter, [1517] develop and improve the social force model to some extent. Reference [18] puts forward cellular automata model for the first time; it is fancied by most scholars because of its advantage in simulating the microscopic behavior of pedestrians. With the deepening of research some improved models are put forward, such as two process model [19], Prefixed Probabilities model [20], two floors model [21], and lattice-gas model $[22,23]$. In addition to the social model and cellular automata model, [24] also puts forward magnetic force model, which has the characteristic of not using empirical relationships crowd density and speed, whereas it adopts Coulomb's theorem describing pedestrians as the object in magnetic field. With the development of computer technology, the computer simulation of pedestrian evacuation process becomes possible. It can simulate pedestrians directly in the moving process of the building and record the different time of pedestrians position change and meanwhile calculate the pedestrian evacuation time. There are about 22 pedestrian evacuation models, among them EVACNET [25, 26], Building EXODUS [27, 28], FIRECAM [29], SIMULEX $[30,31]$, and HAZARD $[32,33]$ have received more attention because of their relatively authentic reflection in pedestrians' choice of escape routes and pedestrians' choice evacuation.

In China, the study of pedestrian and evacuation dynamics theory is in early stages, which is mainly focused on pedestrian traffic characteristics in urban traffic environment and the crowd evacuation in large public places or buildings (e.g., a theatre, a stadium, or a shopping mall). In the first part, [34] proposes the centrifugal force model of pedestrian dynamics and simulates for pedestrian movement process; [35] proposes a cellular automata-based alighting and boarding microsimulation model for passengers in Beijing metro stations; $[36,37]$ propose an evacuation time model for passengers in metro platforms from field data by considering crowd density and physical characteristic of stations; [38-40] investigate pedestrian traffic characteristics of Chinese metropolis and get the basic data of pedestrian traffic characteristics and level of service of crossing facilities by establishing model. In the second part, [41] identifies seven methodological approaches (e.g., cellular automata models, lattice gas models, social force models, fluid-dynamic models, agent-based models, game theoretic models, and approaches based on experiments with animals) for crowd evacuation which has been studied over the last decades; [42] presents a new emergency evacuation system based on MultiAgent framework and Geographic Information System (GIS), which can simulate human's typical behaviors in multiexit public place and present the public evacuation process and in-time distribution during emergency; [43-45] investigate the route choice of pedestrians during evacuation under conditions of both good and zero visibility in a classroom and build a microscopic pedestrians model with discrete space representation.

Through analyzing the current research on pedestrian, this paper maintains that the study of pedestrians and evacuation dynamics is more focused on a building (e.g., a theatre, a stadium, or a shopping mall), rather than the UMH. What is more, these models do not consider the queuing delay caused by limite dcapacity in stairs or escalator, gate and China's pedestrian characteristics are ignored. On the basis of summarizing traditional evacuation time models, this paper proposes to do research, respectively, on the character of the UMHs structure and Chinese pedestrian traffic characteristics and meanwhile use queuing theory and fluid mechanics simulation theory to establish a pedestrian evacuation time model in UMHs, which includes the pedestrian drop-off time, pedestrian evacuation time in platform, and pedestrian evacuation time in the channel.

\section{Experiment Survey Based on Multiple Video Sequences}

\subsection{Experiment Design}

(1) The Selection of Experimental Observation Time and Place. The morning peak and evening peak are different to some extent. Through the survey in the UMHs, the pedestrian traffic volume in the evening peak is higher than the morning peak; the trip by the metro is a commuter trip more often; by contrast, the peak hour mainly focuses on the on and off duty and business travel at the normal working day. The survey time should choose the time in the tremendous traffic demand. Hence, the normal working days are chosen. In addition, through analysis of the pedestrian flow characteristics in the UMHs, business people travel mainly by elastic travel, office people and living people mainly by rigid travel. At the same time, the analysis period should be selected in the maximum hour to analyze in the UMHs. Eventually, we determine the survey period is evening peak. The time of experiment survey based on multiple video sequences is chosen in 17:00-19:00 on the working day and sums up to 2 hours in the evening peak. Moreover, for the purpose of eliminating the influence of human factors, the measurement of geometric parameters in the UMHs is selected at the time of fewer flow. Through the live measurements on the UMHs and analysis of the pedestrian flow characteristic, coupled with the fact where there is only a transfer hub site in Xian, that is, Bei Da-jie station, at the end, we select the Bei Da-jie station as the best observation in the experiment survey, as shown in Figure 1.

(2) The Specific Design Scheme. According to the Bei Da-jie site's CAD drawings of facility layout and the investigation, the position of video cameras is determined, as shown in Figure 2 (the circle is the video camera location); the multiple video sequences can be obtained in the video cameras. In the experiment survey, there are 21 video cameras in the Bei Da-jie station, which record the evolution process of multiple infrastructure in the evening peak.

A specific operation is as follows.

(1) At the exit: we select the video cameras, which represent the purple circle1, the purple circle2, the purple circle3, the purple circle4, and the purple circle5, to observe the pedestrian flow's parameters. 


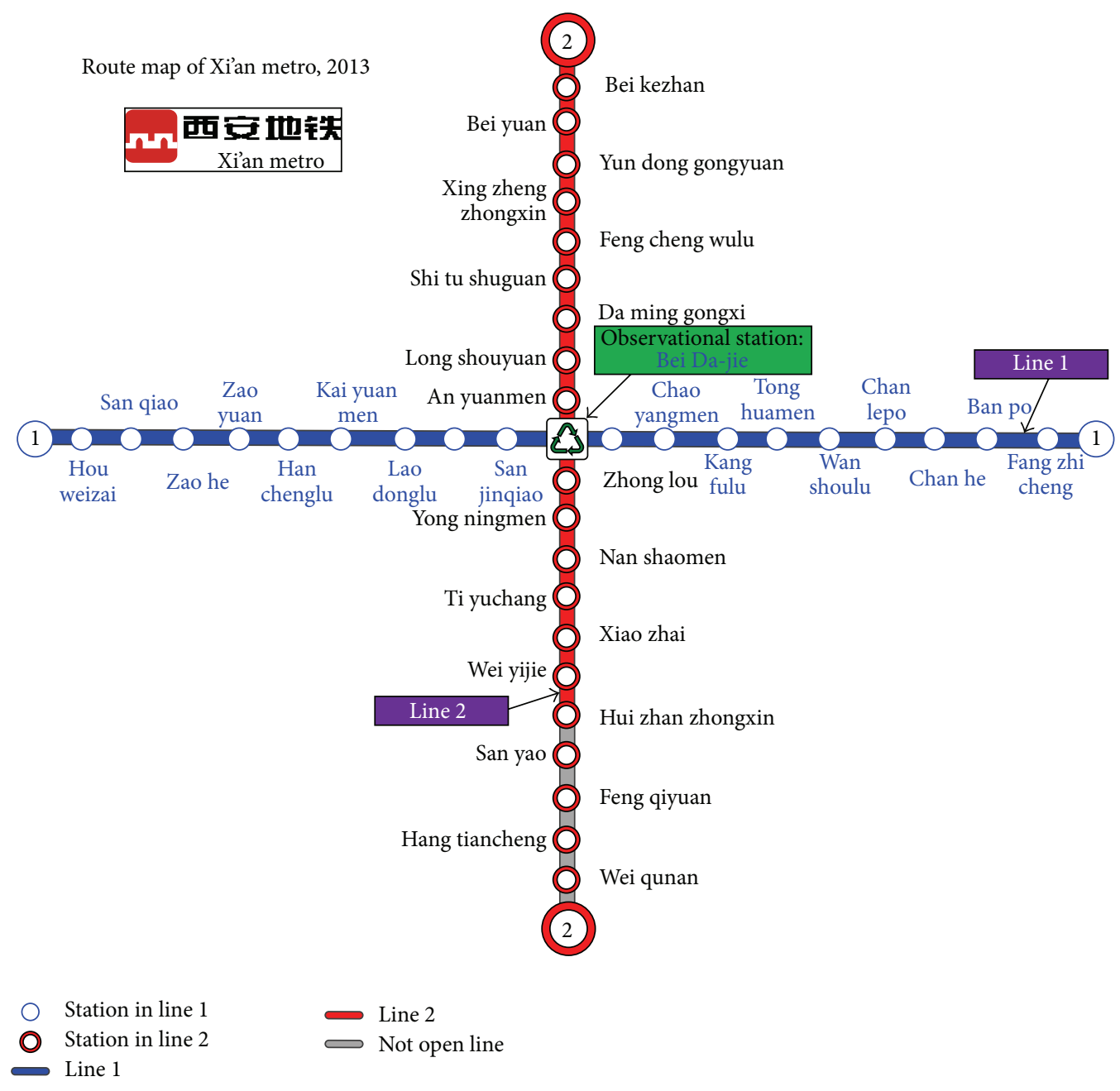

FIgURE 1: Observational station (Bei Da-jie) for pedestrian flow.

(2) On the stairs and escalators: we select the video cameras, which represent the blue circlel, the blue circle2, the blue circle3, the blue circle4, the blue circle5, and the blue circle6, to observe the pedestrian flow's parameters. Where the blue circlel, the blue circle2, and the blue circle 3 are used to observe the parameters of down direction of the stairs and escalators, meanwhile, the blue circle4, the blue circle5, and the blue circle6 are used to observe the parameters of up direction of the stairs and escalators.

(3) In the TVM: we select the video cameras, which represent the green circle1, the green circle2, the green circle3, and the green circle 4 to observe the pedestrian flow's parameters.

(4) In the auto gate: we select the video cameras, which represent the red circlel, the red circle2, the red circle3, and the red circle4 to observe the pedestrian flow's parameters.
(5) In the transfer channel: we select 1 video camera independently to observe the pedestrian flow's parameters in the transfer channel.

(6) On the platform: we select 1 video camera independently to observe the pedestrian flow's parameters in the platform.

\subsection{Data Collecting and Data Processing}

(1) Data Collecting. In order to obtain the pedestrian traffic characteristics exactly in the Bei Da-jie hub, we use the video camera method to obtain the multiple video sequences data, to record video information to collect the pedestrian flow parameter data, which is shown in Figure 3.

Video acquisition method, which is one of the widely methods in the investigation. The advantage of the method is convenient for storage and revisit detail information. At the same time, the advantage of the method is almost collects all of the multiple video sequence data in the pedestrian flow characteristics. The observation pedestrian flow is used to the video acquisition method such as speed, and density in 


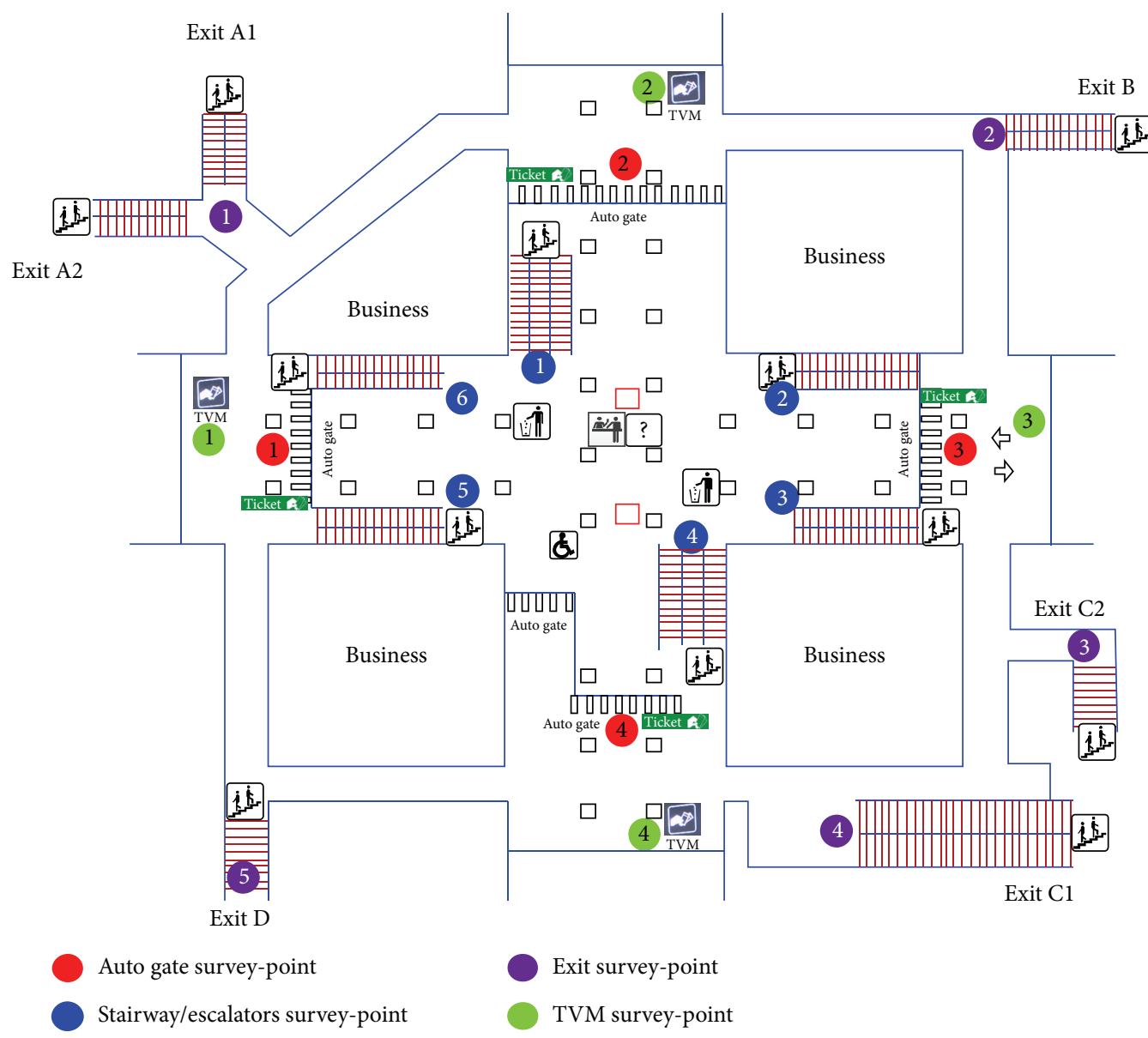

FIgURE 2: The video camera layout drawing in Bei Da-jie hub.

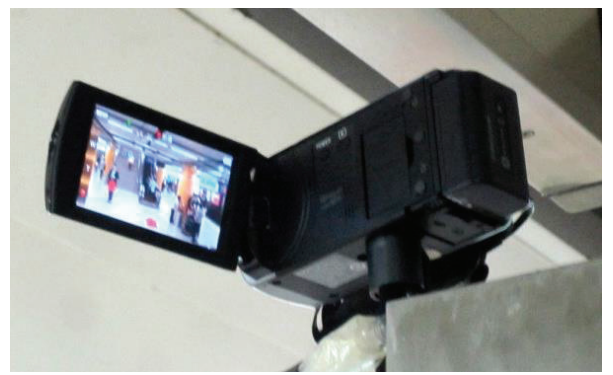

(a)

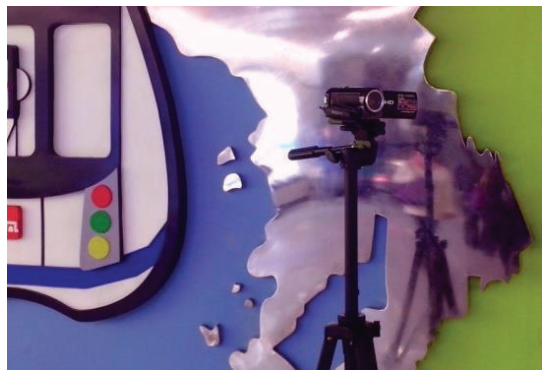

(b)

FIGURE 3: Data collection by the video camera.

the platforms, transfer channels, auto gates, stairs and escalators, exits, and so on.

(2) Data Processing. According to the multiple video sequence data from the camera, every video is divided into framesin a second and play video frame by frame in processing, then, we record the correct time in the observation area between the pedestrian getting into the observation area and leaveing observation area.
The pedestrian speed is calculated by the length and time of the observation area in the camera. In the observation, not only record the speed but also the pedestrian density and the corresponding pedestrian flow of the moment, and then put all the multiple video data into the tables, for the purpose of subsequent data analysis. In the process of data analysis, we use the SPSS to fit the curve relationship of pedestrian flow parameters, and we also test the fitting degree using the value of $R$-squared. 


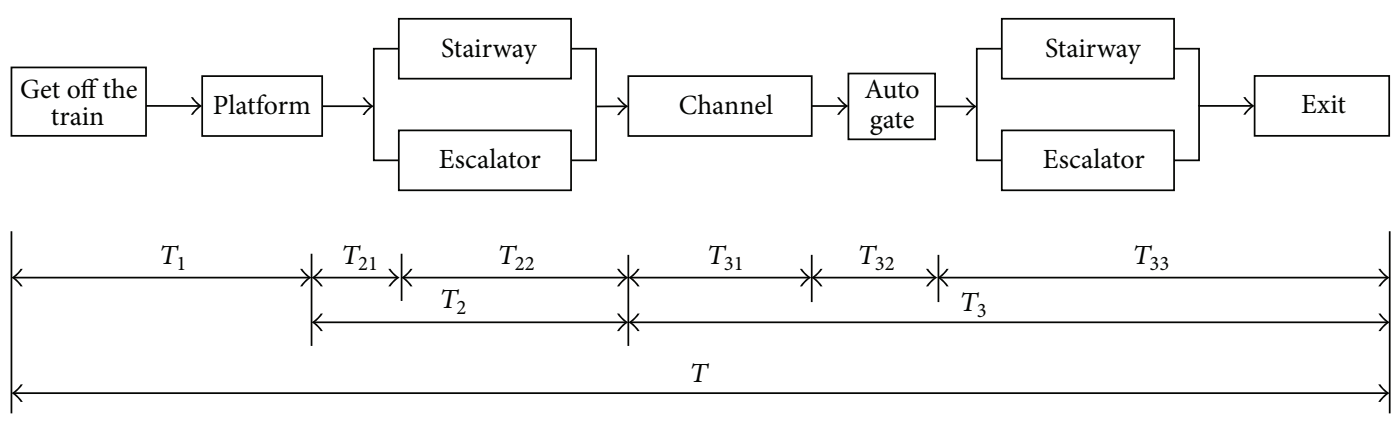

FIgURE 4: Decomposition process of safety evacuation in UMHS.

\section{Pedestrian Evacuation Time Model for UMHS}

Through the observation and analysis in Xian Bei Da-jie hub, the walking speed and spaces characteristic are different from each other; based on these differences between them, we decompose the evacuation process, and the pedestrian evacuation time $(T)$ is divided into three parts; the first part is pedestrian drop-off time $\left(T_{1}\right)$, the second part is safety evacuation time in platform $\left(T_{2}\right)$, and the third part is time of pedestrian pass channel, stairway (or escalators) $\left(T_{3}\right)$. Safety evacuation time is shown in Figure 4, and detailed description for every part of evacuation time is shown as follows.

3.1. Pedestrian Drop-Off Time Model. Pedestrian drop-off time refers to the train arriving at the station stably and the time all passengers droping off from the train to the platform. Drop-off time is related to drop-off numbers, getup numbers, and the width of the train gate door. Here we suppose the pedestrian drop-off time is $T_{1}(s)$.

Through the analysis, we can conclude that the relationship between the single door drop-off time and the number of passengers is different; it can be power exponent relationship, linear relationship, exponential relationship, and natural logarithm. Among them, the power exponent relationship's correlation coefficient $\left(R^{2}\right)$ is the biggest, with the value 0.9924, as shown in Figure 5.

In Figure 5, the number of passengers and drop-off time satisfy the following formula:

$$
T_{1}=\alpha_{1} x_{\max }^{\beta_{1}}=0.6311 x^{0.9884},
$$

where $x_{\max }$ : the largest passengers of drop-off passenger of gate (person), $\alpha_{1}, \beta_{1}$ : Parameters for calibration.

3.2. Pedestrian Evacuation Time in Platform. Safety evacuation time in platform is described in metro design code in China; namely, the width of the exit stairs and evacuation corridor should guarantee the passengers both on the train and the platform and staffs working in the station to escape the fire disaster in 6 minutes. Specific safety evacuation time in platform in metro specification can be seen as follows:

$$
T_{0}=1+\frac{\left(Q_{1}+Q_{2}\right)}{\left\{0.9 \cdot\left[A_{1} \cdot(N-1)+A_{2} \cdot B\right]\right\}} .
$$

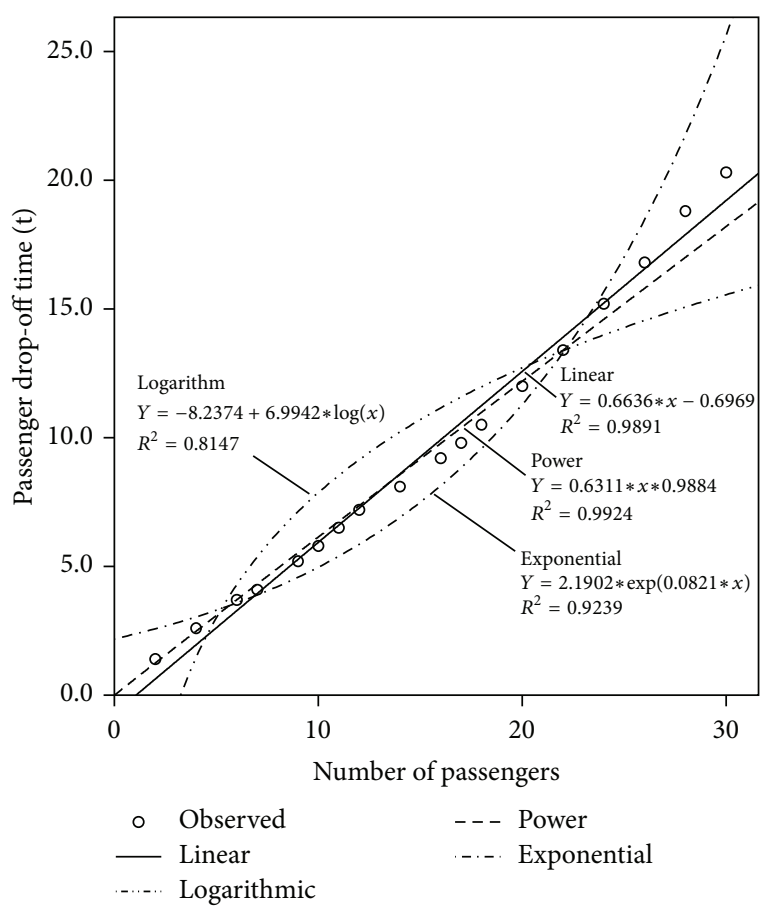

FIGURE 5: The function curve fitting between passenger number and drop-off time.

The evacuation time in platform in metro specification above only considers the biggest evacuation capacity of different facilities and thinks little of the pedestrian density and the impact of environment on pedestrian speed, and the influence of guideline information (directional signs, direction signs, radio evacuation command, radio evacuation message, etc.) is also insightful and thinks little about the factors that may affect traffic organization in the transport hub, making the large deviation time between the theoretical calculation result and the actual evacuation. Hence, in the process of channel (stairway, escalators) evacuation, we consider the speed-density change law of the traffic flow, taking wave theory as an example to simulate the pedestrian flow, as well as considering the accumulation and dissipation characteristic of pedestrian, compared to only considering the evacuation capability on the platform which has improved, further to improve the traditional evacuation time model. 


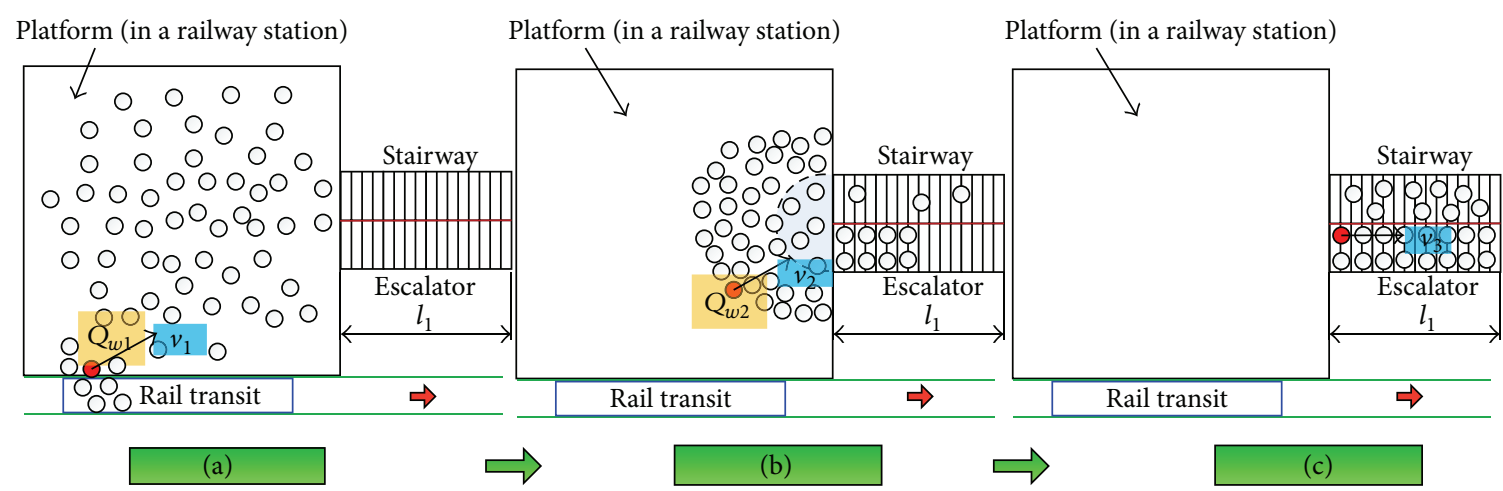

FIgURe 6: Pedestrian evacuation process.

Platform evacuation time can be divided into three phases, as shown in Figure 6. Here we suppose the final pedestrian as the research object, as shown in the red circle in the figure. The final pedestrian (red circle) of the evacuation process can be divided into two stages. The first stage is shown in Figures 6(a) and 6(b), at this period of time, the speed of him is mainly influenced by the evanescent wave which transmits from high density to low density, and the evanescent wave is beginning to transform into the gathering wave. During the forward process, there will be a speed mutation point; the pedestrian (red circle) will suddenly slow down at this point, which is due to the pedestrian waiting in line in front of the evacuation platform, so the flow density is very large and the corresponding speed is very low; the second stage is shown in Figure 6(b) to Figure 6(c), pedestrian (red circle) is mainly affected by the fluctuation of the pedestrian flow, and the gathering wave is beginning to transform into evanescent wave.

Here we suppose the safety evacuation time in platform is $T_{2}(s)$, pedestrian passing time to the stairway (or escalators) is $T_{21}(s)$, and pedestrian passing time in stairway (or escalators) is $T_{22}(s)$. Thus the safety evacuation time mode in the platform is shown below

$$
\begin{gathered}
Q_{w 1}=\frac{\left(v_{1}-v_{2}\right)}{\left(1 / k_{1}-1 / k_{2}\right)}, \\
Q_{w 2}=\frac{\left(v_{3}-v_{2}\right)}{\left(1 / k_{3}-1 / k_{2}\right)}, \\
T_{21}=T_{1} \cdot \frac{Q_{w 1}}{\left(Q_{w 1}-Q_{w 2}\right)},
\end{gathered}
$$

where $Q_{w 1}$-wave flow of pedestrians aggregation wave after getting off $(\mathrm{p} /(\mathrm{m} \cdot \mathrm{s})), Q_{w 2}$ - wave flow of pedestrians evanescent wave on stairway (or escalator) $(\mathrm{p} /(\mathrm{m} \cdot \mathrm{s})), v_{1}$ pedestrian normal walking speed after getting off the train $(\mathrm{m} / \mathrm{s}), v_{2}$-pedestrian dissipation speed on stairway (or escalators) $(\mathrm{m} / \mathrm{s}), v_{3}$-escalator running speed or pedestrians speed climbing up the stairway $(\mathrm{m} / \mathrm{s}), k_{1}$-pedestrian density under $v_{1}$ state $\left(\mathrm{p} / \mathrm{m}^{2}\right), k_{2}$-pedestrian density under $v_{2}$ state $\left(\mathrm{p} / \mathrm{m}^{2}\right)$, and $k_{3}$-pedestrian density under $v_{3}$ state $\left(\mathrm{p} / \mathrm{m}^{2}\right)$ :

$$
T_{22}=\frac{l_{1}}{v_{3}}
$$

where $l_{1}$-stairway (or escalator) length $(\mathrm{m})$.

Thus the evacuation time in platform $T_{2}$ is

$$
T_{2}=T_{21}+T_{22}
$$

3.3. Pedestrian Evacuation Time in Channel (Stairway, Escalators). We suppose the time of pedestrian pass channel, stairway (or escalators), is $T_{3}(s)$, the time of pedestrian pass channel is $T_{31}(s)$, pedestrian dissipation time on auto gate is $T_{32}(s)$, and pedestrians pass time on stairway (or escalators) is $T_{33}(s)$.

(1) Calculation of the Time of Pedestrian Pass Channel. Pedestrian speed in the corridor or stairway is largely influenced by flow density. The pedestrians occupied smaller place when the passenger flow volume is larger, and the pedestrian speed is slower. While on the other hand, the smaller the flow density, the faster the pedestrian speed. According to the collected data in the Bei Da-jie station, we find the relationship between speed and density in pass channel, which is shown in Figure 7.

According to the fitting functions between the speed and density of pedestrians, we can see that there is a strong correlation between the speed and density of pedestrians in a subway corridor or in the stairway (upward direction and downward direction of the stairs), and they comply with third-degree polynomial curve $\left(R^{2}\right.$ is the biggest, with the value 0.934 ), which is shown in Table 1.

Thus the time of pedestrian pass channel can be shown as follows:

$$
T_{31}=\frac{l_{2}}{v(k)}=\frac{l_{2}}{\left(\alpha_{2} k^{3}+\beta_{2} k^{2}+\gamma k+C\right)},
$$

where $l_{2}$-channel length $(\mathrm{m}), k$-pedestrian density in corridor ( $\mathrm{p} / \mathrm{m} 2)$, and $\alpha_{2}, \beta_{2}, \gamma, C$ - parameters for calibration.

(2) Calculation of Pedestrian Dissipation Time on Auto Gate. Here we suppose the mean arrival rate of pedestrian flow is $\lambda$ and the average service rate of auto gate is $\mu$; thus the traffic intensity of auto gate is $\rho$. And we assume that the time of the pedestrian pass through the auto gate meets the standard $\mathrm{M} / \mathrm{M} / 1$ model (Figure 8 ).

In the standard $\mathrm{M} / \mathrm{M} / \mathrm{C}$ and $\mathrm{M} / \mathrm{M} / \mathrm{1} / \mathrm{C}$ model, the regulation of characteristics of those models is the same with 
TABLE 1: Model summary and parameter estimates.

\begin{tabular}{lccccccrrr}
\hline \multirow{2}{*}{ Equation } & \multicolumn{4}{c}{ Model summary } & \multicolumn{4}{c}{ Parameter estimates } \\
& R Square & $F$ & df1 & df2 & Sig. & Constant & $b 1$ & $b 2$ & $b 3$ \\
\hline Logarithmic & 0.795 & 236.71 & 1 & 61 & 0 & 1.228 & -0.355 & -0.113 \\
Cubic & 0.934 & 276.576 & 3 & 59 & 0 & 1.651 & -0.229 & 0.022 \\
Exponential & 0.894 & 516.655 & 1 & 61 & 0 & 1.836 & -0.349 & \\
\hline
\end{tabular}

The independent variable: density.

Dependent variable: speed.

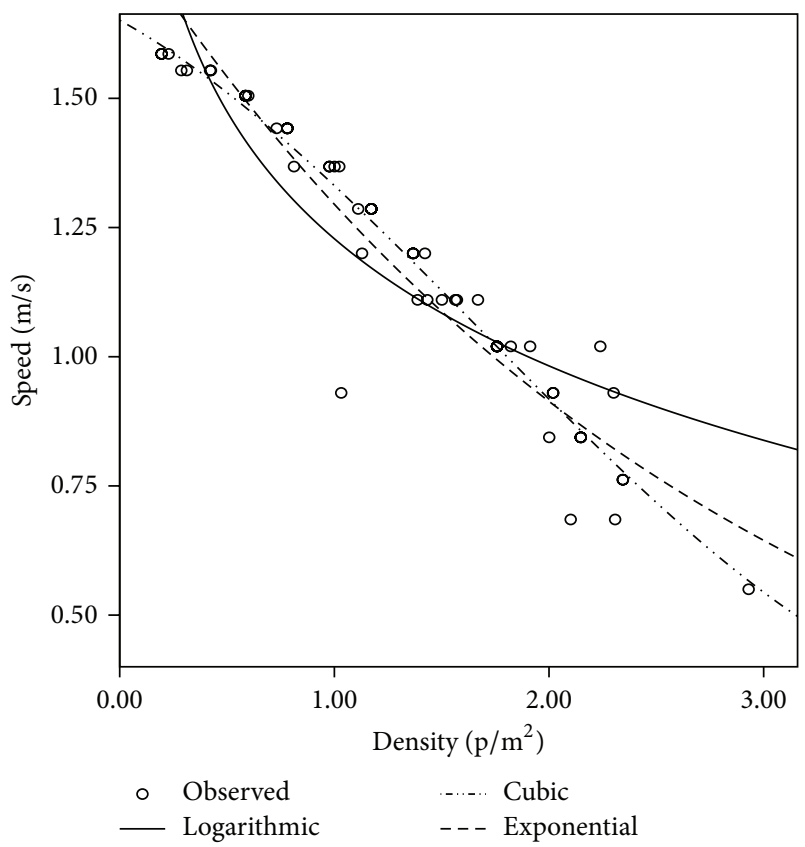

FIgURE 7: Create fit curve between speed and density.

the standard $M / M / 1$. In addition, specified the auto gates are mutual independent and the average service rates are the same; namely, $\mu_{1}=\mu_{2}=$ the $\mu_{c}=\mu$. So the average service rate for the whole services agency is $c \mu(n \geqslant c)(n \mu(n<c))$.

Similarly, we find

(a) the entire gates idle probability

$$
P_{0}=\left[\sum_{k=1}^{c-1} \frac{1}{k !}\left(\frac{\lambda}{\mu}\right)^{k}+\frac{1}{c !} \cdot \frac{1}{1-\rho} \cdot\left(\frac{\lambda}{\mu}\right)^{c}\right]^{-1},
$$

(b) average number of customers in system $L_{s}$

$$
L_{s}=L_{q}+\frac{\lambda}{\mu}
$$

(c) average numbers of customer in the queue $L_{q}$

$$
L_{q}=\sum_{n=c+1}^{\infty}(n-c) P_{n}=\frac{(c \rho)^{c} \rho}{c !(1-\rho)^{2}} P_{0}
$$

(d) expected value of stay time in the system

$$
W_{s}=\frac{L_{s}}{\lambda}
$$

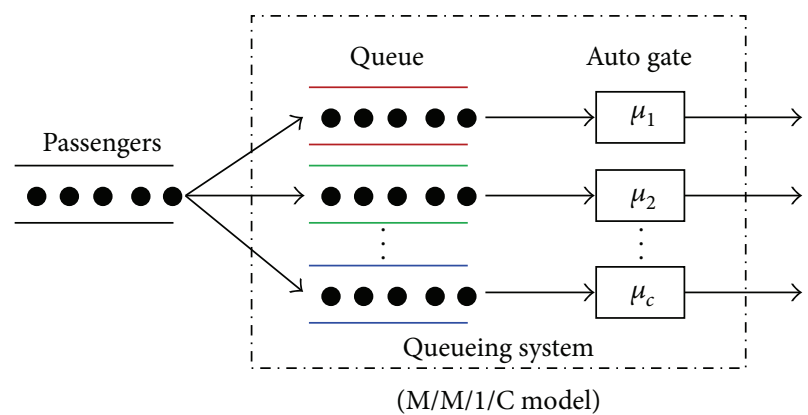

FIGURE 8: Passengers queuing at the auto gate.

Thus, pedestrian dissipation time on auto gate is $T_{32}$

$$
T_{32}=W_{s} \text {. }
$$

(3) Calculation of Pedestrians Pass Time on Stairs (or Escalators). For escalator, the speed and width of it are fixed, and the pedestrians are upward and downward with it automatically without walking on it, so the time is basically fixed. As shown in the following:

$$
T_{33}=\frac{l_{1}}{v_{3}},
$$

where $l_{1}$-stairway (or escalator) length $(\mathrm{m})$.

The whole pedestrian evacuation time in the Urban Metro Hub is as follows:

$$
T_{3}=T_{31}+T_{32}+T_{33}
$$

3.4. Pedestrian Evacuation Time Model. The safety evacuation time of pedestrian is divided into three parts, and with those three parts added up we can get the whole evacuation time. Taking the pedestrian drop-off time, safety evacuation time in platform, time of pedestrian pass channel, and stairway (or escalators) into consideration, the safety evacuation time model in UMHs is established and is shown in what follows:

$$
T=\sum_{i=1}^{3} T_{i}
$$

\subsection{Example Verification}

3.5.1. General Situation. In this paper, we take the Bei Dajie metro hub in Xi'an as an example, mainly based on two 
TABLE 2: Observed values of model parameters in various stages.

\begin{tabular}{|c|c|c|c|c|}
\hline Number & Model name & Index name & Observed values & Unit \\
\hline 1 & Pedestrian drop-off time & $T_{1}$ & 20 & - \\
\hline \multirow{7}{*}{2} & \multirow{7}{*}{ Pedestrian evacuation time in platform } & $v_{1}$ & 1.495 & $(\mathrm{~m} / \mathrm{s})$ \\
\hline & & $v_{2}$ & 1.05 & $(\mathrm{~m} / \mathrm{s})$ \\
\hline & & $v_{3}$ & 0.93 & $(\mathrm{~m} / \mathrm{s})$ \\
\hline & & $k_{1}$ & 1.167 & $\left(\mathrm{p} / \mathrm{m}^{2}\right)$ \\
\hline & & $k_{2}$ & 1.193 & $\left(\mathrm{p} / \mathrm{m}^{2}\right)$ \\
\hline & & $k_{3}$ & 2.181 & $\left(\mathrm{p} / \mathrm{m}^{2}\right)$ \\
\hline & & $l_{1}$ & 28.24 & $\mathrm{~m}$ \\
\hline \multirow{5}{*}{3} & \multirow{5}{*}{ Pedestrian evacuation time in channel } & $k$ & 1.314 & $\left(\mathrm{p} / \mathrm{m}^{2}\right)$ \\
\hline & & $l_{2}$ & 100 & $\mathrm{~m}$ \\
\hline & & $c$ & 6 & - \\
\hline & & $\mu$ & 0.5 & $(\mathrm{p} / \mathrm{min})$ \\
\hline & & $\lambda$ & 1.35 & $(\mathrm{p} / \mathrm{min})$ \\
\hline
\end{tabular}

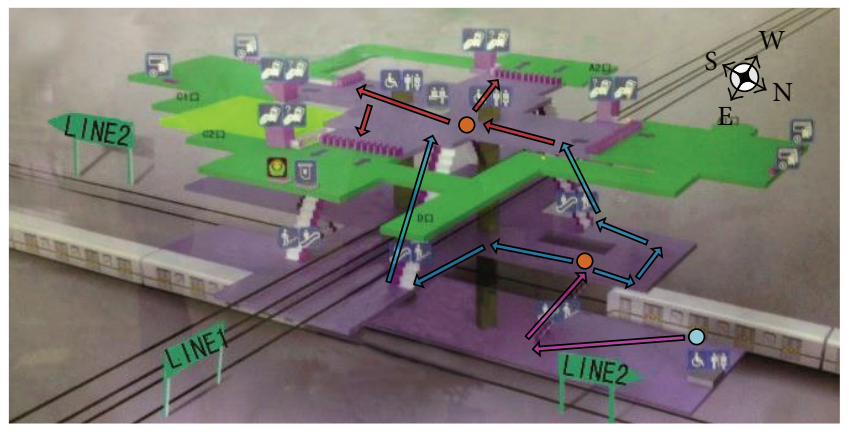

\begin{abstract}
$\Longleftarrow$ Pedestrian flow in B3F
$\Longleftarrow$ Pedestrian flow in $\mathrm{B} 2 \mathrm{~F}$

$\Longleftarrow$ Pedestrian flow in $\mathrm{B} 1 \mathrm{~F}$
\end{abstract}

FIgURE 9: Pedestrians' walking flow lines.

reasons. On the one hand, the station is a typical "ten" shaped metro interchange station, line 1 and line 2 meet here, line 1 site is side platform, line 2 site is an island platform, and the pedestrians' walking flow lines are shown in Figure 9. On the other hand, the station is the only staggered floor transfer station based on a ladder in Xian, which is characterized by the shortest distance traveled by passengers; especially in peak hours the transfer time is not more than one minute. Therefore, a higher request for passenger transport organization and train time interval coordinate should be put forward.

3.5.2. The Relevant Data Measured. Before studying the pedestrians' transfer time model in Xian Bei Da-jie station, some parameters are measured, which are shown in Table 2.

\subsubsection{Results}

(1) The Observed Results. Transferring the case to the Bei Dajie when the peak hours of pedestrian flow is short, and a cycle of pedestrian flow evacuation time is measured; each stage
TABLE 3: The observed pedestrian evacuation time in each stage.

\begin{tabular}{lc}
\hline Stage name & Observed time (s) \\
\hline Pedestrian drop-off time & 11.16 \\
Pedestrian evacuation time in platform & 44.86 \\
Pedestrian evacuation time in channel & 113.85 \\
Total time & $\mathbf{1 6 9 . 8 7}$ \\
\hline
\end{tabular}

TABLE 4: The calculated pedestrian evacuation time in each stage.

\begin{tabular}{lc}
\hline Stage name & Calculated time (s) \\
\hline Pedestrian drop-off time & 12.23 \\
Pedestrian evacuation time in platform & 42.76 \\
Pedestrian evacuation time in channel & 111.64 \\
Total time & $\mathbf{1 6 6 . 6 4}$ \\
\hline
\end{tabular}

of the pedestrian evacuation time is shown in Table 3. The total number of pedestrians is 317 that transfer to line 1 from line 2 , the last pedestrian used 172.82 seconds totally when he arrived at line 1 platform.

(2) The Calculated Results. The established evacuation time model in this paper is used to calculate each stage of the pedestrian evacuation time; the result is shown in Table 4.

(3) Comparison Analysis. Comparing the observed time with the calculated time, we can see that the error between them is only $1.90 \%$. What is more, the error in each stage is also very tiny; the result is shown in Table 5 . In the end, the estimation results of the established model in the paper and measured values are consistent; the applicability of the model is reasonable.

\section{Conclusions}

(1) Through calculating the whole evacuation time in Xian Bei Da-jie transfer hub, the observed results in the model are $169.87 \mathrm{~s}$, the calculated results in the model are $166.64 \mathrm{~s}$ as well, and the relative error between them is $1.90 \%$, where 
TABLE 5: The relative error analysis.

\begin{tabular}{lccc}
\hline Stage name & Observed time $(\mathrm{s})$ & Calculated time $(\mathrm{s})$ & Relative error \\
\hline Pedestrian drop-off time & 11.16 & 12.23 & $-9.59 \%$ \\
Pedestrian evacuation time in platform & 44.86 & 42.76 & $4.68 \%$ \\
Pedestrian evacuation time in channel & 113.85 & 111.64 & $1.94 \%$ \\
Total time & $\mathbf{1 6 9 . 8 7}$ & $\mathbf{1 6 6 . 6 4}$ & $\mathbf{1 . 9 0 \%}$ \\
\hline
\end{tabular}

we have the following. (a) The pedestrian drop-off time's measured value is $11.16 \mathrm{~s}$, the value of calculation is $12.23 \mathrm{~s}$, and the relative error is $-9.59 \%$. (b) The value of measure in platform evacuation time is $44.86 \mathrm{~s}$, the value of calculation is $42.76 \mathrm{~s}$, and the relative error is $4.68 \%$. (c) The value of measure in channel evacuation time is $113.85 \mathrm{~s}$, the value of calculation is $111.64 \mathrm{~s}$, and the relative error is $1.94 \%$. Compared to the Code for Design of Metro (GB 50157003) promulgated by Ministry of Housing and Urban-Rural Construction of the People's Republic of China (MOHURD), the whole time is $166.64 \mathrm{~s}$, less than the $6 \mathrm{~min}$ in Code for Design of Metro (GB 50157-003), and the applicability of the pedestrian evacuation time model was verified.

(2) In terms of the pedestrian drop-off time model, through fitting analysis with the multiple video sequence data, the four functions of the relationship are fitted between passengers getting off them through a single door and a number of alighting passengers; the fitting functions are composed of the exponent relationship, linear relationship, exponential relationship, and the natural logarithm relationship. The correlation coefficient of exponent relationship is the largest and the correlation coefficient $\left(R^{2}\right)$ is 0.9924. The relationship between passengers getting off time and the number of alighting passengers is an exponent relationship. For the platform evacuation time model, the pedestrian evacuation process obeys the wave theory. As an improvement, compared to Code for Design of Metro (GB 50157-003), we consider the pedestrian flow speed-density changing laws and combining with the flow wave theory, the platform evacuation time model has been improved for better reflection of the pedestrian flow distribution characteristics in this paper.

(3) In terms of the channel evacuation time models, the pedestrian evacuation time model has been split into the time that pedestrians pass through the corridor, the auto gate, and stairs or escalators. The research result indicates that there is a strong quartic polynomial relation between pedestrian flow velocity and density of the subway corridor and upward direction and downward direction of the stairs. The time of pedestrians through the auto gate is to meet the standard M/M/C model. The actual evacuation time in the hub corridor can be better reflected while comprehensively considering the time, which consider pedestrians passing through the corridor, the auto gate, and stairs or escalators into the channel evacuation time model.

(4) In addition, as a future work, we only take the Bei Da-jie transfer hub in Xi'an as an example and verify the feasibility of the proposed pedestrian evacuation time model. Next, we should do lots of experiment surveys, such as other Urban Metro Hubs in Xi'an, verify and modified the model. In addition, could the Urban Metro Hubs (UMHs) become a full-time laboratory? What if you could analyze every transaction, capture insights from every passenger interaction, and did not have to wait for months to get data from the field? What if...? Big data are flooding in at rates never seen before-doubling every 18 months. In the future, how to set up the platform for big data, big discovery, and big decision in the UMHs? The answer is technology. Technology for capturing and analyzing big data is widely available at ever-lower price points. With the recent developments of sensing, networking, and computing technologies, more and more UMHs-related big data and computational resources become available.

\section{Conflict of Interests}

The authors declare that there is no conflict of interests regarding the publication of this paper.

\section{Acknowledgements}

This paper has been supported by the National Natural Science Foundation of China (Grant no. 51208051) and the Fundamental Research Funds for the Central Universities (CHD2011ZD014). The authors deeply appreciate the supports. The authors wish to acknowledge the contribution of all the expert groups and other team members, including Associate Professor Yong-gang WANG, Dr. Long-fei WANG, Dr. Xiao-wei LI, and M.E. Yu-ting HE. The authors also wish to express their gratitude to the anonymous reviewers for their insightful comments and suggestions to an earlier version of the paper. Special gratitude is extended to those construction academics and industry practitioners who have responded to and contributed their valuable input to complete the survey questionnaires.

\section{References}

[1] L. F. Henderson, "The statistics of crowd fluids," Nature, vol. 229, no. 5284 , pp. $381-383,1971$.

[2] L. F. Henderson, "On the fluid mechanics of human crowd motion," Transportation Research, vol. 8, no. 6, pp. 509-515, 1974.

[3] L. F. Henderson and D. J. Lyons, "Sexual differences in human crowd motion," Nature, vol. 240, no. 5380, pp. 353-355, 1972.

[4] D. Helbing, "A mathematical model for the behavior of individuals in a social field," Journal of Mathematical Sociology, vol. 19, pp. 189-219, 1994. 
[5] S. J. Yuhaski Jr. and J. M. Smith, "Modeling circulation systems in buildings using state dependent queueing models," Queueing Systems, vol. 4, no. 4, pp. 319-338, 1989.

[6] D. Garbrecht, Describing Pedestrian and Car Trips by Transition Matrices, vol. 27 of Traffic Quarterly, 1973.

[7] A. J. Mayne, "Some further results in the theory of pedestrians and road traffic," Biometrika, vol. 41, pp. 375-389, 1954.

[8] A. Borgers and H. Timmermans, "A model of pedestrian route choice and demand for retail facilities within inner-city shopping areas," Geographical Analysis, vol. 18, no. 2, pp. 115$128,1986$.

[9] M. Asano, T. Iryo, and M. Kuwahara, "Microscopic pedestrian simulation model combined with a tactical model for route choice behaviour," Transportation Research C, vol. 18, no. 6, pp. 842-855, 2010.

[10] D. Helbing, "A fluid dynamic model for the movement of pedestrians," Complex Systems, vol. 6, pp. 391-415, 1998.

[11] D. Helbing, L. Buzna, A. Johansson, and T. Werner, "Selforganized pedestrian crowd dynamics: experiments, simulations, and design solutions," Transportation Science, vol. 39, no. 1, pp. 1-24, 2005.

[12] D. Helbing, I. J. Farkas, P. Molnar, and T. Vicsek, "Simulation of pedestrian crowds in normal and evacuation situations," Pedestrian and Evacuation Dynamics, vol. 21, pp. 21-58, 2002.

[13] D. Helbing, P. Molnár, I. J. Farkas, and K. Bolay, "Self-organizing pedestrian movement," Environment and Planning B, vol. 28, no. 3, pp. 361-383, 2001.

[14] D. Helbing and T. Vicsek, "Optimal self-organization," New Journal of Physics, vol. 1, pp. 13.1-13.17, 1999.

[15] D. R. Parisi and C. O. Dorso, "Microscopic dynamics of pedestrian evacuation," Physica A, vol. 354, no. 1-4, pp. 606-618, 2005.

[16] K. Teknomo, "Application of microscopic pedestrian simulation model," Transportation Research F, vol. 9, no. 1, pp. 15-27, 2006.

[17] A. Seyfried, B. Steffen, and T. Lippert, "Basics of modelling the pedestrian flow," Physica A, vol. 368, no. 1, pp. 232-238, 2006.

[18] T. Toffoli and N. H. Margolus, "Invertible cellular automata: a review," Physica D, vol. 45, no. 1-3, pp. 229-253, 1990.

[19] V. J. Blue and J. L. Adler, "Cellular automata microsimulation for modeling bi-directional pedestrian walkways," Transportation Research B, vol. 35, no. 3, pp. 293-312, 2001.

[20] F. Weifeng, Y. Lizhong, and F. Weicheng, "Simulation of bi-direction pedestrian movement using a cellular automata model," Physica A, vol. 321, no. 3-4, pp. 633-640, 2003.

[21] C. M. Henein and T. White, "Macroscopic effects of microscopic forces between agents in crowd models," Physica A, vol. 373, pp. 694-712, 2007.

[22] X. Guo, J. Chen, S. You, and J. Wei, "Modeling of pedestrian evacuation under fire emergency based on an extended heterogeneous lattice gas model," Physica A, vol. 392, pp. 1994-2006, 2013.

[23] X. Guo, J. Chen, Y. Zheng, and J. Wei, "A heterogeneous lattice gas model for simulating pedestrian evacuation," Physica A, vol. 391, no. 3, pp. 582-592, 2012.

[24] S. Okazaki and S. Matsushita, "A study of simulation model for pedestrian movement with evacuation and queuing," in Proceedings of the International Conference on Engineering for Crowd Safety, pp. 271-280, 1993.

[25] T. M. Kisko, R. Francis, and C. Nobel, Evacnet4 User's Guide, University of Florida, 1998.
[26] A. T. Syed and M. A. Hassanain, "A simulation model for emergency evacuation time of a library facility using EVACNET4," Structural Survey, vol. 31, pp. 1-10, 2013.

[27] E. R. Galea and J. M. Perez Galparsoro, "A computer-based simulation model for the prediction of evacuation from masstransport vehicles," Fire Safety Journal, vol. 22, no. 4, pp. 341366, 1994.

[28] L. Yang, P. Rao, K. Zhu, S. Liu, and X. Zhan, "Observation study of pedestrian flow on staircases with different dimensions under normal and emergency conditions," Safety Science, vol. 50, no. 5, pp. 1173-1179, 2012.

[29] D. Yung, G. Hadjisophocleous, and G. Proulx, "A description of the probabilistic and deterministic modelling used in FiRECAM," International Journal on Engineering Performance-Based Fire Codes, vol. 1, pp. 18-26, 1999.

[30] R. Fahy, "A practical example of an evacuation model for complex spaces," in Proceedings of the Human Behaviour in Fire1st International Symposium, pp. 743-751, 1999.

[31] C. Rogsch and W. Klingsch, "Basics of software-tools for pedestrian movement-identification and results," Fire Technology, vol. 48, no. 1, pp. 105-125, 2012.

[32] N. T. N. Anh, Z. J. Daniel, N. H. Du, A. Drogoul, and V. D. An, "A hybrid macro-micro pedestrians evacuation model to speed up simulation in road networks," in Advanced Agent Technology, vol. 7068, pp. 371-383, Springer, 2012.

[33] P. A. Thompson and E. W. Marchant, "A computer model for the evacuation of large building populations," Fire Safety Journal, vol. 24, no. 2, pp. 131-148, 1995.

[34] W. J. Yu, R. Chen, L. Y. Dong, and S. Q. Dai, "Centrifugal force model for pedestrian dynamics," Physical Review E, vol. 72, no. 2, Article ID 026112, 2005.

[35] Q. Zhang, B. Han, and D. Li, "Modeling and simulation of passenger alighting and boarding movement in Beijing metro stations," Transportation Research C, vol. 16, no. 5, pp. 635-649, 2008.

[36] S.-K. Chen, S.-Y. Li, X. Li, J. Hong, J.-X. Lai, and P. Cai, "Modeling evacuation time for passengers from metro platforms," Journal of Transportation Systems Engineering and Information Technology, vol. 8, no. 4, pp. 101-107, 2008.

[37] S.-K. Chen, S. Liu, X. Xiao, J. Hong, and B.-H. Mao, "M/G/c/cbased model of passenger evacuation capacity of stairs and corridors in metro stations," Journal of the China Railway Society, vol. 34, no. 1, pp. 7-12, 2012.

[38] S. Feng, N. Ding, T. Chen, and H. Zhang, "Simulation of pedestrian flow based on cellular automata: a case of pedestrian crossing street at section in China," Physica A, vol. 392, pp. 28472859, 2013.

[39] B. X. Song, Z. Z. Wu, and Z. H. Xie, "Study on occupant evacuation model considering influence of direction sign," China Safety Science Journal, vol. 12, pp. 27-33, 2011.

[40] L.-X. Yang, X.-M. Zhao, Z.-Y. Gao, and J.-F. Zheng, "Bidirectional pedestrian flow model with traffic convention," Acta Physica Sinica, vol. 60, no. 10, Article ID 100501, 2011.

[41] X. Zheng, T. Zhong, and M. Liu, "Modeling crowd evacuation of a building based on seven methodological approaches," Building and Environment, vol. 44, no. 3, pp. 437-445, 2009.

[42] Y. Bo, W. Yong-gang, and W. Cheng, "A multi-agent and GIS based simulation for emergency evacuation in park and public square," in Proceedings of the IEEE International Conference on Computational Intelligence and Security (CIS '09), pp. 211-216, 2009. 
[43] R. Y. Guo and H. J. Huang, "Theoretical analysis and simulation of pedestrian evacuation under invisible conditions," Simulation, vol. 88, pp. 1138-1148, 2012.

[44] R.-Y. Guo, H.-J. Huang, and S. C. Wong, "Route choice in pedestrian evacuation under conditions of good and zero visibility: experimental and simulation results," Transportation Research B, vol. 46, pp. 669-686, 2012.

[45] Y. Xu and H.-J. Huang, "Simulation of exit choosing in pedestrian evacuation with consideration of the direction visual field," Physica A, vol. 391, no. 4, pp. 991-1000, 2012. 


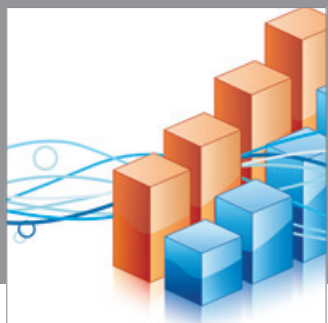

Advances in

Operations Research

mansans

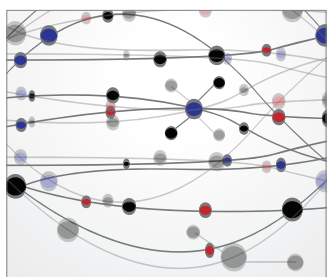

The Scientific World Journal
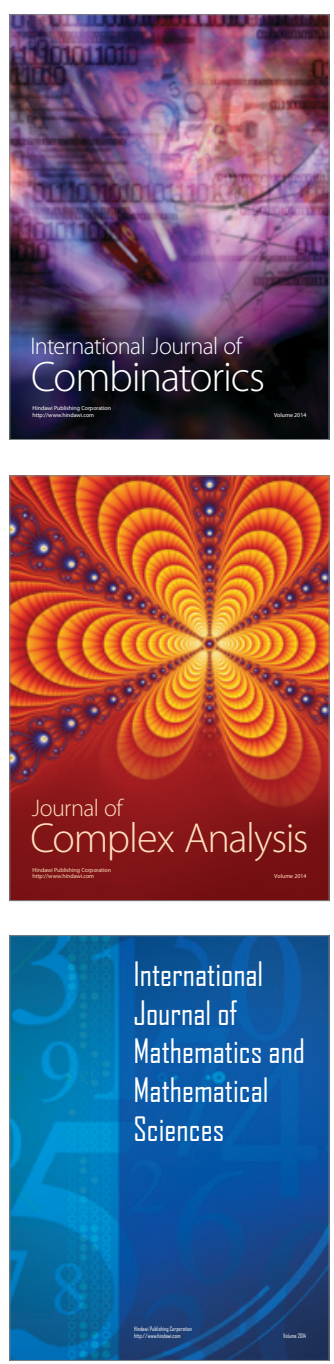
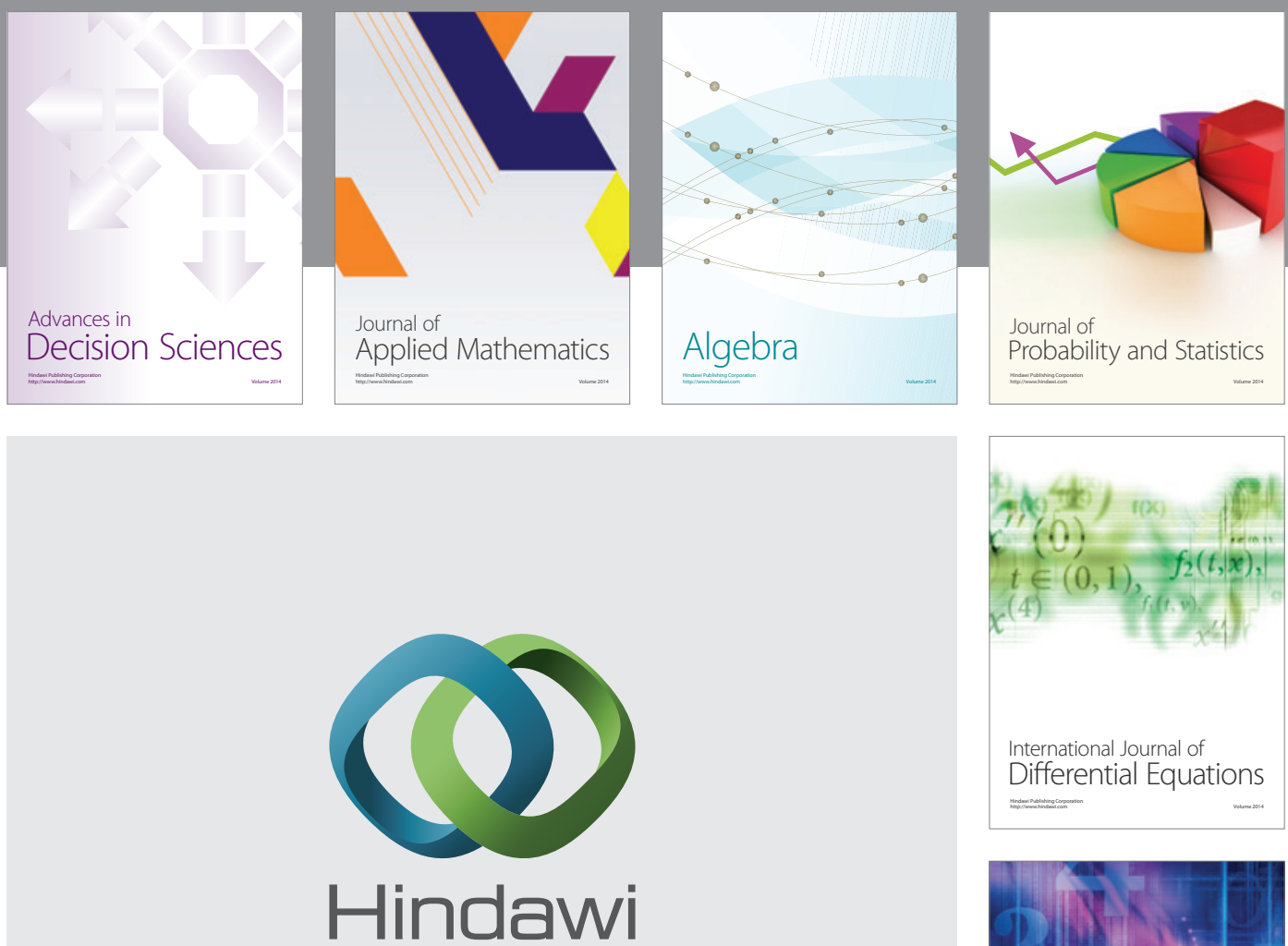

Submit your manuscripts at http://www.hindawi.com
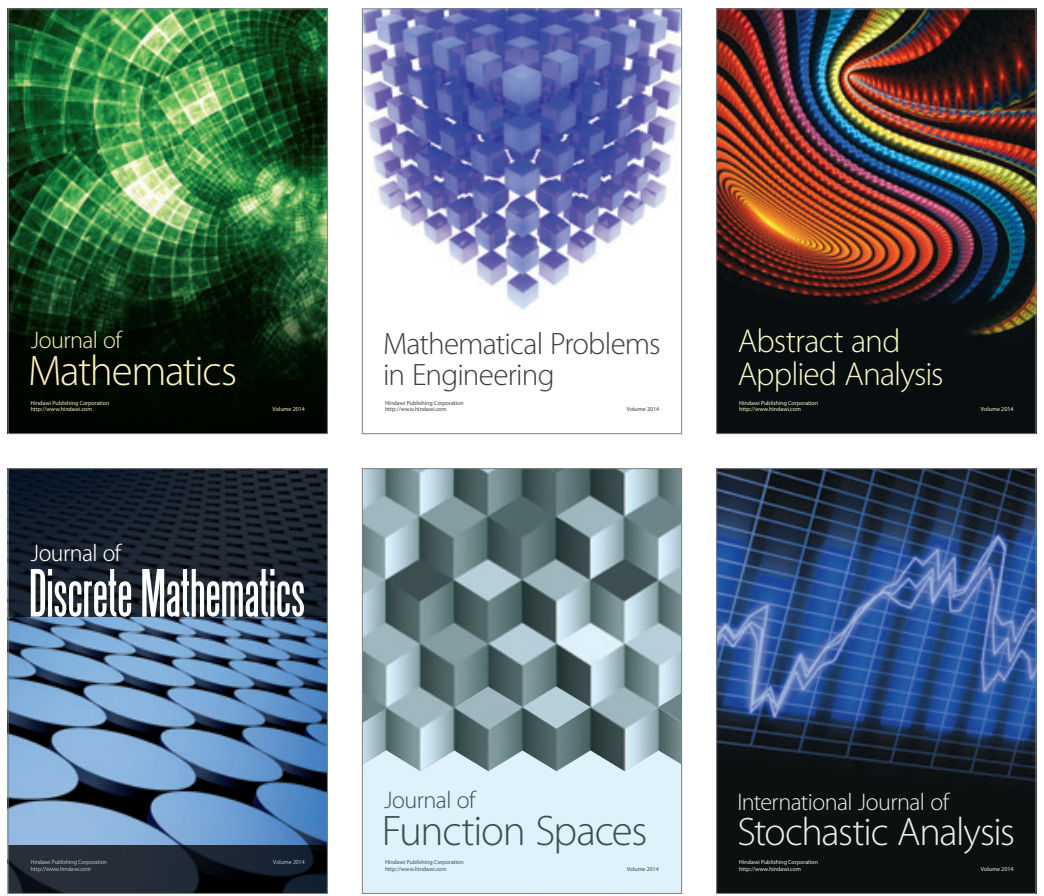

Journal of

Function Spaces

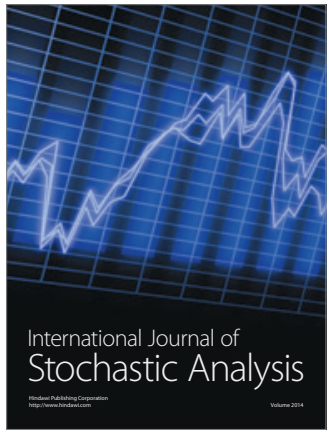

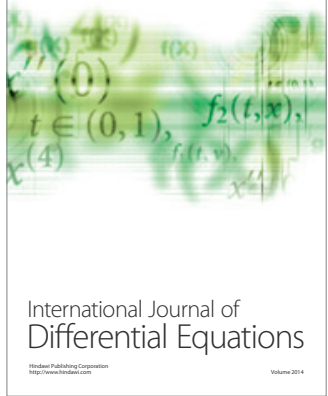
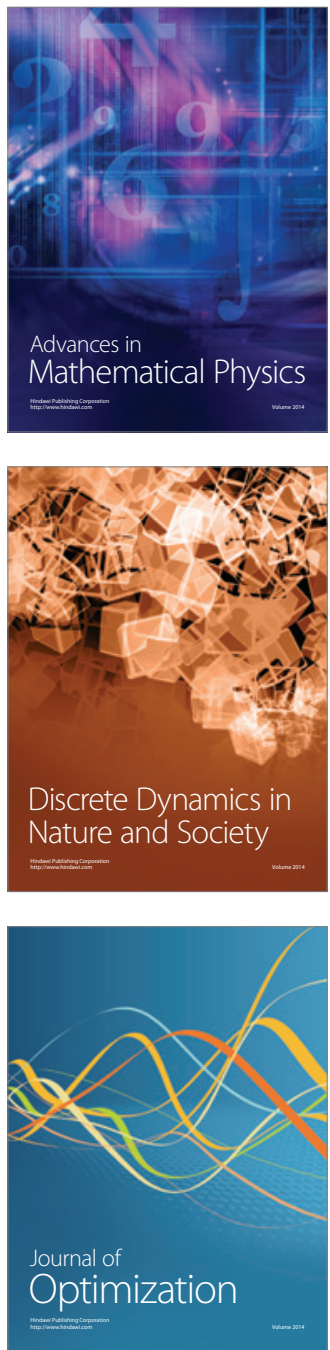